OPEN ACCESS

Edited by:

Shanchun Guo,

Xavier University of Louisiana,

United States

Reviewed by:

Peng Wang,

Sun Yat-sen University, China

Mamunur Rashid,

Columbia University, United States

${ }^{*}$ Correspondence:

Ji Wang

doctorwang2009@126.com

Qi Wang

wangqi710@126.com

†These authors have contributed equally to this work

Specialty section:

This article was submitted to

Molecular Medicine,

a section of the journal

Frontiers in Cell and Developmental

Biology

Received: 01 December 2020

Accepted: 19 January 2021

Published: 09 February 2021

Citation:

Zhou Y, Chen X, Zheng Y, Shen R, Sun S, Yang F, Min J, Bao L, Zhang $Y$, Zhao X, Wang J and Wang Q (2021) Long Non-coding RNAs and mRNAs Expression Profiles of Monocyte-Derived Dendritic Cells

From PBMCs in AR.

Front. Cell Dev. Biol. 9:636477. doi: 10.3389/fcell.2021.636477

\title{
Long Non-coding RNAs and mRNAs Expression Profiles of Monocyte-Derived Dendritic Cells From PBMCs in AR
}

\author{
Yumei Zhou 1t, Xuemei Chen ${ }^{1 t}$, Yanfei Zheng ${ }^{1 t}$, Rongmin Shen', Shuxian Sun', Fei Yang', \\ Jiayu Min', Lei Bao', Yan Zhang ${ }^{1}$, Xiaoshan Zhao'2, Ji Wang ${ }^{1 *}$ and Qi Wang ${ }^{1 *}$
}

${ }^{1}$ National Institute of TCM Constitution and Preventive Medicine, School of Chinese Medicine, Beijing University of Chinese Medicine, Beijing, China, ${ }^{2}$ School of Traditional Chinese Medicine, Southern Medical University, Guangzhou, China

Objective: The objective of this study is to explore the long non-coding RNAs (IncRNAs) and messenger RNAs (mRNAs) expression profiles of monocyte-derived dendritic cells (DCs) obtained from peripheral blood mononuclear cells (PBMCs). DCs are known to play a major role in the regulating function of allergic rhinitis (AR).

Methods: PBMCs were separately isolated from the human peripheral blood of patients with AR and normal person (NP). The mixed lymphocyte reaction (MLR) assay was used to evaluate the function of DCs. Flow cytometry was used to determine the immune regulatory function of immature DCs (imDCs) and mature DCs (mDCs). IncRNAs and mRNAs in the NP group (DCs isolated from NP) and the test group (DCs isolated from patients with AR) were identified via chip technology and bioinformatic analyses. Moreover, bioinformatic analyses were employed to identify the related biological functions of monocyte-derived DCs and construct the functional networks of IncRNAs and mRNAs that are differentially expressed (DE) in imDCs and mDCs.

Results: MLR was significantly higher in the mDCs group than that in the imDCs group. CD14 was highly expressed in imDCs, whereas HLA-DR, CD80, and CD86 were highly expressed in mDCs $(p<0.001)$. We identified 962 DE IncRNAs and 308 DE mRNAs in the imDCs of NP and patients with AR. Additionally, there were 601 DE IncRNAs and 168 DE mRNAs in the mDCs in the NP and test groups. Quantitative RT-qPCR was used to study the significant fold changes of IncRNAs and mRNAs. The Kyoto Encyclopedia of Genes and Genomes (KEGG) analysis found 16 significant regulated pathways in imDCs and 10 significant regulated pathways in mDCs, including the phagosome, cell adhesion signaling pathway, and inflammatory mediator regulation of TRP channels pathway.

Conclusion: Our research studied the IncRNA and mRNA expression profiles of monocyte-derived DCs and demonstrated the functional networks that are involved in monocyte-derived DCs-mediated regulation in AR. These results provided possible molecular mechanisms of monocyte-derived DCs in the immunoregulating function and laid the foundation for the molecular therapeutic targets of AR. 


\section{BACKGROUND}

Allergic rhinitis (AR) is a very common allergic disease that affects $10-40 \%$ of the global population (Bousquet et al., 2008). Its remarkable prevalence and relapses put an extensive burden on its patients and the society. Furthermore, AR negatively impacts the quality of life of patients with AR. There had been a marked increase in the prevalence of AR during the past years (Wang et al., 2016). AR turns into asthma if it is not treated in time, and the adequate treatment of AR can alleviate the severity of asthma (Leynaert et al., 2004). Researchers have illustrated that mast cell infiltration, lymphocytes imbalance, and goblet cell hyperplasia are involved in the pathogenesis of AR (Ouyang et al., 2010; Poggi et al., 2012). AR, which is a type I allergic disorder that is mediated by IgE humoral immune response, is accompanied by an influx of eosinophils and $\mathrm{T}$ helper 2 cells that secrete pro-inflammatory cytokines, namely, IL-4, IL-5, and IL-13 (Wilson et al., 2005). Abnormal innate and adaptive immune responses play a major role in the pathogenesis of AR.

Dendritic cells (DCs), which are the most important antigen-presenting cells (APCs) that send signals to the $T$ cells, mainly participate in the pathogenesis of many diseases with immunoregulatory mechanisms, such as AR. DCs link the innate and adaptive immune responses. The peripheral blood mononuclear cells (PBMCs) have a round nucleus (Delves, 2016). PBMCs include lymphocytes, monocytes, and DCs. In humans, the frequencies of these DCs vary among individuals. PBMCs are divided into various functional subtypes with respect to the specific cytokine expression profiles, surface markers, and the transcription factors. Phenotypic and functional assessments of PBMC research lay the foundation of the human immune system research; hence, the knowledge that population is represented in the peripheral blood and how they act with other immune cells is essential. Additionally, the results from human PBMC studies (Schiekofer et al., 2003; Tacconi et al., 2004; Chang et al., 2014) cannot be neglected. Therefore, it is important to know the progression of $\mathrm{AR}$ along with its expression profiles in PBMCs, especially DCs.

Long non-coding RNAs (lncRNAs), over $200 \mathrm{nt}$ in length, is a type of RNA that does not a protein coding function (Ulitsky and Bartel, 2013). These RNAs have been regarded as indispensable epigenetic regulators and are probably involved in the cell's biological behaviors (Kopp and Mendell, 2018). For example, they are involved in regulating the homeostasis of the immune system (Wang et al., 2014; Du et al., 2017). However, it is critical to find out whether IncRNA can immunoregulate DC in the progression of AR.

The combination of lncRNA-messenger RNA (mRNA) expression profiles and functional networks is adopted to analyze the DC-mediated regulation functions. These results improve our understanding of lncRNAs in the immunoregulatory function of monocyte-derived DCs and indicate the potential targets for the curative treatment of AR.

\section{MATERIALS AND METHODS}

\section{Subjects}

Patients with AR visited doctors in the outpatient service in the Guo Yi Tang of Beijing University of Chinese Medicine. In this study, there were 24 subjects: 12 males and 12 females. They were divided into two groups: the AR group (patients with AR, 12 subjects: five males and seven females) and NP group (normal persons, 12 subjects: four males and eight females). With support/approval from the Ethics Committee of Beijing University of Chinese Medicine, this study was conducted while adhering to the principles of the Declaration of Helsinki. Patients in the AR group were positive for skin puncture test, including pollen, food, dust mites, paint, or molds as well as in specific IgE. Two weeks before study recruitment, these patients with $\mathrm{AR}$ received no topical or systemic corticosteroid therapy. We chose the study participants with no history of smoking or other immune system disorders, such as rheumatoid arthritis, systemic lupus erythematosus, and scleroderma.

\section{Isolation of PBMCs and Generation of DCs}

The whole blood samples obtained from the two groups were stored in vacuum tubes with heparin, and PBMCs were isolated from these samples by lymphocyte separation solution (Tianjin Haoyang Biological Manufacture Co., Ltd.). Mononuclear cells were seeded in 12-well plates with the RPMI 1640 medium that contains $10 \%$ heat-inactivated fetal calf serum (FCS, GIBCO, Germany) and $2 \mathrm{mM}$ of L-glutamine (R10 medium, Sigma, St. Louis, MO, United States). After incubation at $37^{\circ} \mathrm{C}$ for $2 \mathrm{~h}$, the non-adherent cells were removed and the adherent cells were cultured within the medium containing $100 \mathrm{ng} / \mathrm{ml}$ of $\mathrm{rhGM}$ CSF and $100 \mathrm{ng} / \mathrm{ml}$ of rhIL-4 (R\&D Systems, Minneapolis, MN, United States). On the sixth day, $50 \mathrm{ng} / \mathrm{ml}$ of TNF- $\alpha$ (R\&D Systems, Minneapolis, MN, United States) was added into the samples; the method had the same protocol in the study of Andreia et al. (2005). Immature DCs (imDCs) were collected on the fifth day, and the mature DCs (mDCs) were collected on the seventh day.

\section{Mixed Lymphocyte Reaction (MLR)}

After being treated with $25 \mu \mathrm{g} / \mathrm{mL}$ of mitomycin at $37^{\circ} \mathrm{C}$ for $30 \mathrm{~min}$, the DCs were placed at the concentrations of $2 \times 10^{8}$ cells per well at a quantity of $200 \mu \mathrm{l}$ and incubated with non-adherent PBMCs obtained from the same healthy people. These samples were later stimulated by lymphocytes in the concentration proportions of 1:10, 1:50, and 1:100. Thereafter, the samples were mixed and incubated with nonadherent PBMC from the same healthy persons at the same concentrations in triplicate. The cells were treated with 10\% fetal bovine serum. Then, $10 \mu \mathrm{l}$ of 3-(4,5-dimethylthiazol-2-yl)2,5-diphenyl-2H-tetrazolium bromide (MTT) solution $(5 \mathrm{mg} / \mathrm{ml}$, medium dilution, Sigma-Aldrich Chemical Co., St. Louis, 
MO, United States) was added to each well, and the cells were incubated for $72 \mathrm{~h}$ in the incubator. Then, $150 \mu \mathrm{l}$ of DMSO was added followed by the addition of enzymes after $4 \mathrm{~h}$. The absorbance was detected using a spectrophotometer at $570 \mathrm{~nm}$.

\section{Surface Marker Expression Analysis in imDCs and $\mathrm{mDCs}$}

The CD14 (PerCP-Cy 5.5, BD Biosciences, United States), HLA-DR (APC, BD Biosciences, United States), and isotype mouse IgG2a-PE (PE, BioLegend, United States) were added in the samples of imDCs. Moreover, CD86-APC, CD80-PE, and isotype mouse IgG1-FITC (BioLegend, United States) were added in the samples of mDCs. The cells were then suspended with precooled PBS, counted under a microscope, and centrifuged at $1000 \mathrm{~g}$ for $5 \mathrm{~min}$. Data were acquired using a FACSCalibur cytometer (BD Biosciences, United States) and the ratios of $\mathrm{CD}_{14}{ }^{+}, \mathrm{HLA}_{-} \mathrm{DR}^{+}, \mathrm{CD}^{+} 0^{+}$, and $\mathrm{CD}^{+}{ }^{+} \mathrm{DCs}$ were determined.

\section{RNA Extraction, Labeling, Chip Hybridization, and Scanning}

The RNA extraction, labeling, chip hybridization, and scanning were all finished following the use of Agilent Human lncRNAmRNA profiling chip ( $4^{*} 180 \mathrm{~K}$, Design ID: 062918). All the chip results were detailed according to the processes of software operations. The screening criteria were to increase or decrease the fold change value $\geq 2.0$ and $p$-value $<0.05$.

\section{Gene Ontology (GO) and Pathway Enrichment Analysis}

The GO and Kyoto Encyclopedia of Genes and Genomes (KEGG) enrichment analysis were used to study the differentially expressed (DE) mRNAs. The results of the target gene analysis of lncRNA and the mRNA expression results on the chip need to be correlated so that the upregulated and downregulated $\mathrm{DE}$ genes can be investigated. The corrected $p$-value $<0.05$ by calculating the FDR and FDR $<0.05$ was selected as the threshold.

\section{LncRNA-mRNA-Weighted Co-expression Network}

The correlation of the IncRNA-mRNA expression in the imDCs and mDCs was calculated. Then, the relationship pairs of LncRNA and mRNA based on the abovementioned criteria ( $p$-value $<0.05$, FDR $<0.05$ ) were screened. The coexpression network of lncRNAs and mRNAs was constructed, and the co-expression network of lncRNAs and mRNAs was then established.

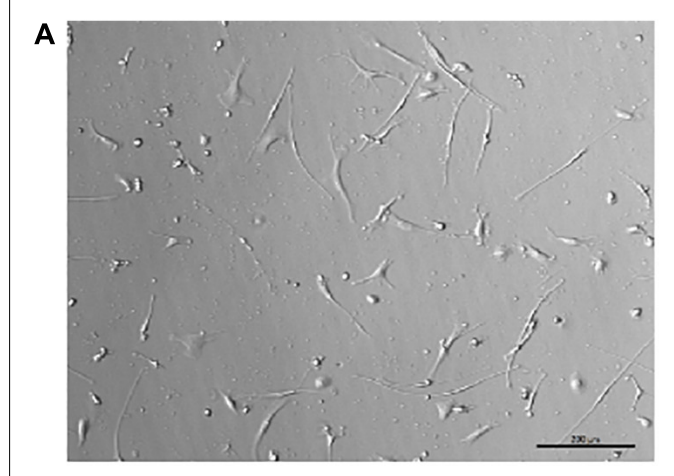

B

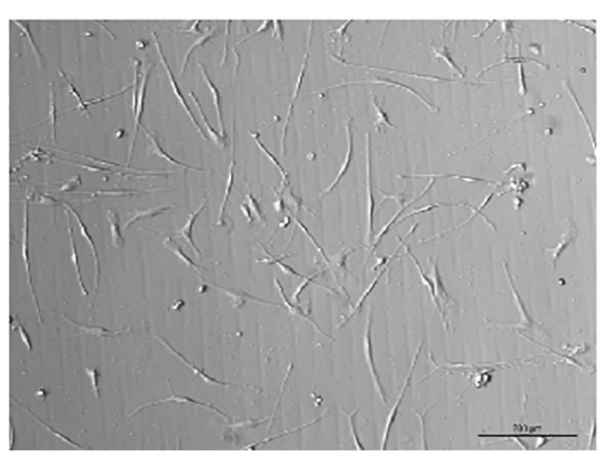

C

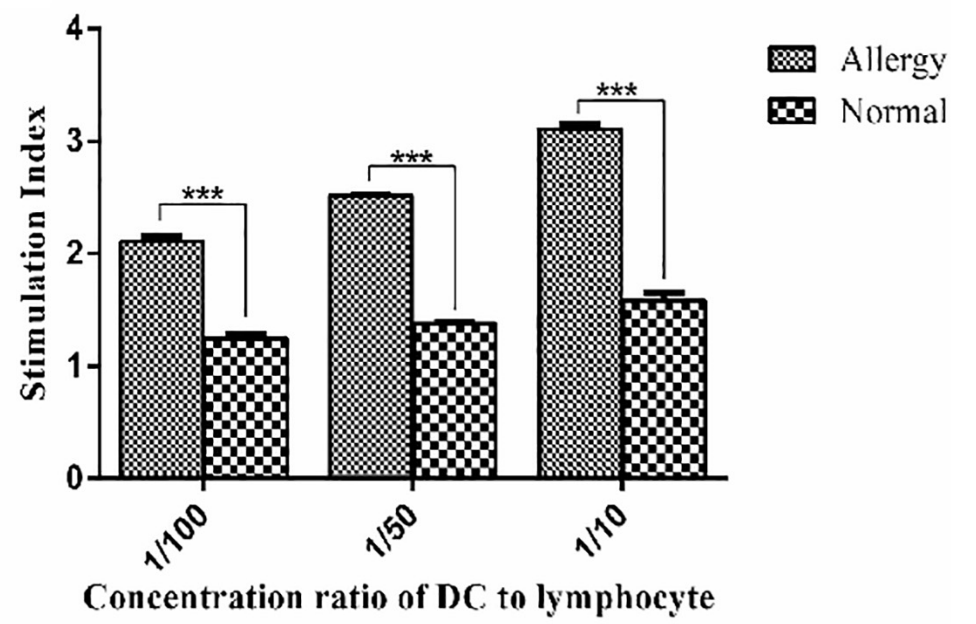

FIGURE 1 | Morphology of dendritic cells and the functional activity of DCs were assayed by autologous MLR. (A,B) imDCs and mDCs, mDCs have a more structure of dendrites. (C) MLR was determined by the stimulation index. $2 \times 10^{8} \mathrm{ml}^{-1} \mathrm{DCs}$ and lymphocytes were cultured with the medium for $72 \mathrm{~h}$, and then DCs were collected and autologous MLR was performed. The control group was stimulated with $25 \mathrm{mg} / \mathrm{L}$ mitomycin as controls. ${ }^{\star \star \star} P<0.001$. 


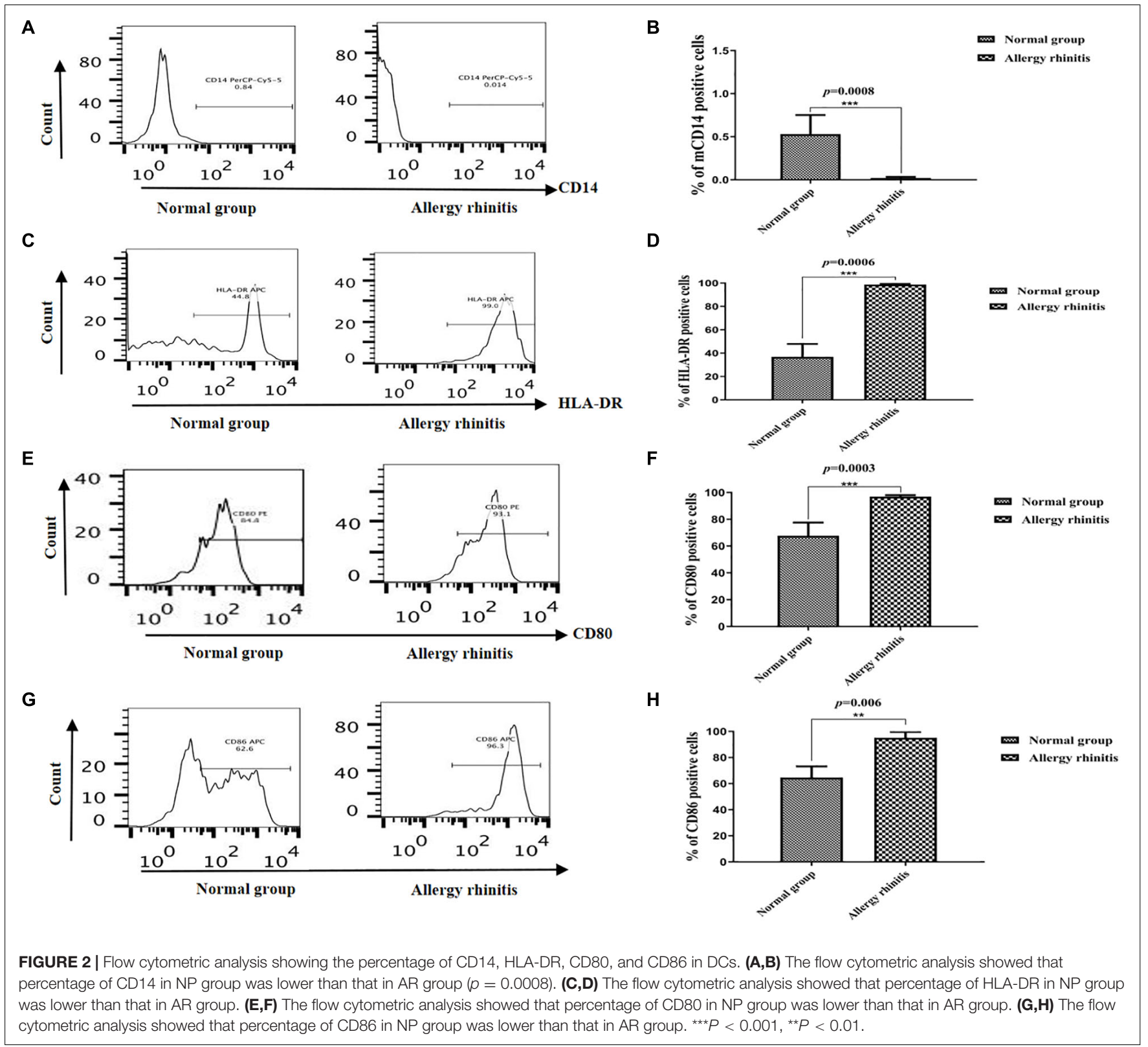

\section{Real-Time PCR Verification of DE Genes}

Quantitative RT-qPCR was used to investigate the different expressions of imDCs' and $\mathrm{mDCs}$ ' genes between the patients with AR and NP. The total RNA was extracted from imDCs and DCs according to the kit for cells. In all, $20 \mu \mathrm{g}$ of total RNA was converted into cDNA by using oligo ( $\mathrm{dT}$ ) and reverse transcriptase (Thermo, United States) to analyze the qPCR results. The thermal cycler conditions were set as follows: amplificated at $95^{\circ} \mathrm{C}$ for $10 \mathrm{~min}, 95^{\circ} \mathrm{C}$ for $15 \mathrm{~s}, 60^{\circ} \mathrm{C}$ for $60 \mathrm{~s}, 40$ cycles of denaturation $\left(15 \mathrm{~s}, 94^{\circ} \mathrm{C}\right), 15 \mathrm{~s}$ at $95^{\circ} \mathrm{C}$, and a combined process of annealing and extension (1 $\mathrm{min}$, $60^{\circ} \mathrm{C}$ ). Supplementary Table S1 shows the primers for these genes.

\section{Statistical Analysis}

The SPSS 22.0 software was used for statistical analysis in this study. Results were expressed as mean \pm standard deviation. The relationship of lncRNAs and mRNAs was determined by the Spearman's correlation coefficient. $p$-values $<0.05$ were considered significant values for this study.

\section{RESULTS}

\section{Allogeneic T-Cell Proliferation Experiment}

In allergenic mixed lymphocyte reaction (MLR), the levels of $\mathrm{T}$-cell proliferation were increased with the proportion of $\mathrm{T}$ cells. 


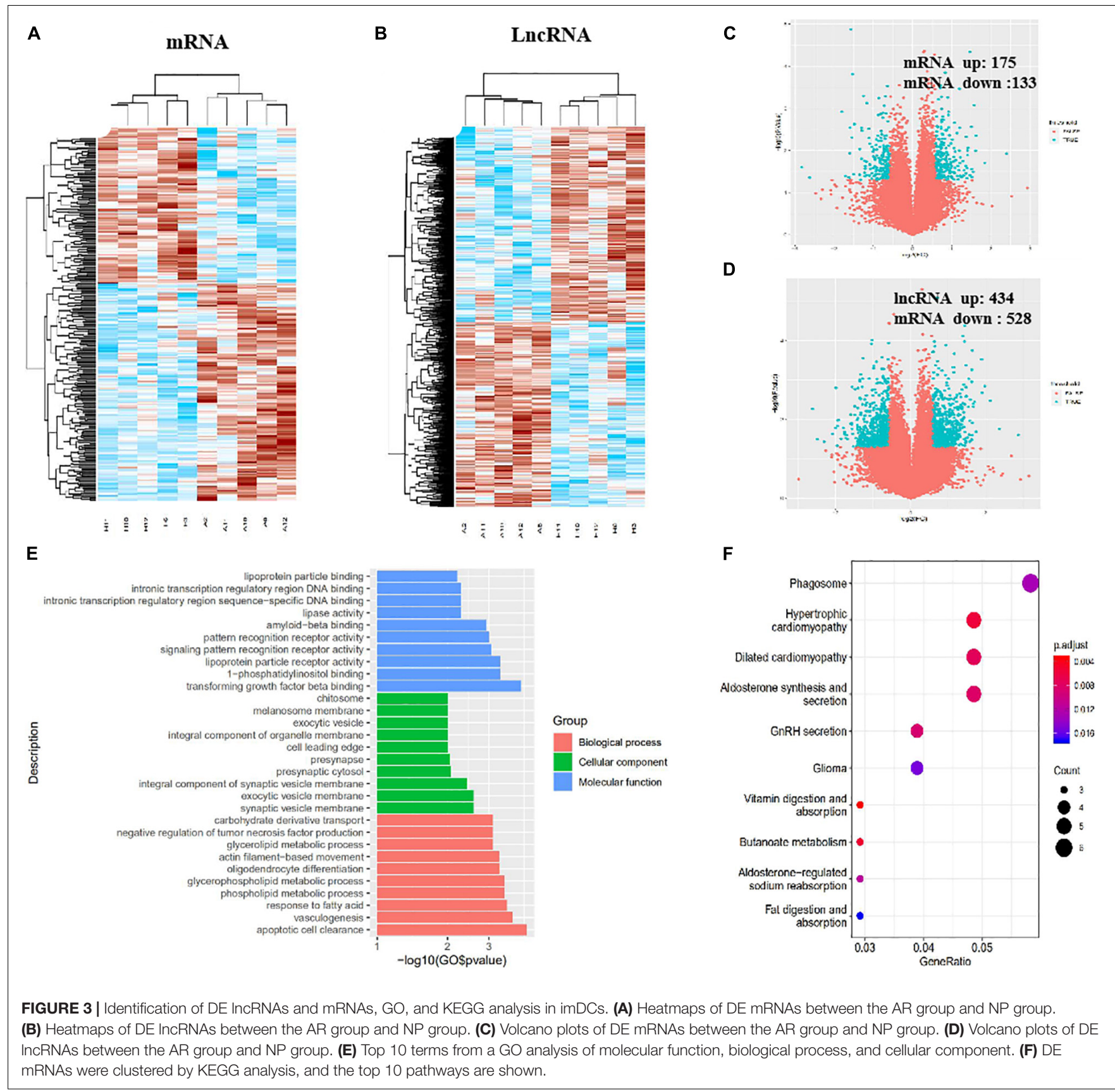

DCs in the patients with AR have a stronger stimulation ability than NP as shown in Figures 1A-C. When the ratio of DC cells to $\mathrm{T}$ cells was 1:50 and 1:100, the difference was significant $(p<0.001)$.

\section{Immunophenotype of DCs in Patients With AR and NP}

We evaluated the percentages of CD14, HLA-DR, CD80, and CD86 in the DCs. The immunophenotypic characteristics of DCs in patients with AR were compared with that of NP, as shown in Figure 2. The CD14 concentration of imDCs was lower in patients with AR than that in the NP group. The mean percentage of $\mathrm{CD} 14^{+} \mathrm{DCs}$ in the patients with AR was $0.84 \pm 0.25 \%$ (median: $0.78 \%$ ), and it was significantly lower than the value in the NP group $(p=0.0008)$, where the mean percentage of these cells was $0.012 \pm 0.013 \%$ (median: $0.017 \%$ ) (Figures 2A,B).

The mean proportion of HLA-DR ${ }^{+}$mDCs in the patients with AR was $44.25 \pm 8.64 \%$ (median: $44.8 \%$ ) and was higher $(p=0.0006)$ than the NP group, where the mean percentage of these cells was $95.3 \pm 3.84 \%$ (median: 99.0\%) (Figures 2C,D).

The mean proportion of $\mathrm{CD}^{+} 0^{+} \mathrm{mDCs}$ in the patients with AR was $72.15 \pm 7.64 \%$ (median: $67.78 \%$ ) and was 
TABLE 1 | The characteristics of mRNAs with the largest fold change in imDCs.

\begin{tabular}{|c|c|c|c|}
\hline GeneName & Genbank Accession & FC (abs) & Regulation \\
\hline Fatty acid 2-hydroxylase & NM_024306 & 2.7454393 & Up \\
\hline Uncharacterized LOC645984 & AK095436 & 2.289749 & Up \\
\hline Shisa homolog 9 (Xenopus laevis) & NM_001145205 & 2.3817623 & Down \\
\hline NK2 homeobox 1 & NM_003317 & 2.2473493 & Down \\
\hline Sodium channel, voltage-gated, type VII, alpha & NM_002976 & 3.4931335 & Down \\
\hline Nucleolar and spindle associated protein 1 & NM_016359 & 2.0980568 & Up \\
\hline Ventral anterior homeobox 1 & NM_199131 & 2.798632 & Down \\
\hline Chromosome 20 open reading frame 132 & NM_213631 & 2.831788 & Up \\
\hline Programmed cell death 1 ligand 2 & NM_025239 & 3.0216887 & Down \\
\hline Solute carrier family 29 (nucleoside transporters), member 1 & NM_001078177 & 2.2991307 & Up \\
\hline Very low density lipoprotein receptor & NM_001018056 & 2.4687624 & Down \\
\hline Tropomyosin 2 (beta) & NM_213674 & 2.175945 & Down \\
\hline Uncharacterized LOC100131129 & AK127184 & 2.1942973 & Up \\
\hline Peptidyl arginine deiminase, type ॥ & NM_007365 & 3.0724247 & Up \\
\hline CD300 molecule-like family member $f$ & NM_139018 & 2.5870113 & Up \\
\hline Programmed cell death 1 ligand 2 & NM_025239 & 2.9215207 & Down \\
\hline CD36 molecule (thrombospondin receptor) & NM_001001547 & 2.167322 & Down \\
\hline Suppression of tumorigenicity 5 & NM_005418 & 2.673464 & Up \\
\hline T-cell acute lymphocytic leukemia 1 & NM_003189 & 2.3056405 & Down \\
\hline Fc receptor-like B & NM_001002901 & 2.3118186 & Up \\
\hline
\end{tabular}

higher $(p=0.0003)$ than the NP group, where the mean percentage of these cells was $95.3 \pm 3.84 \%$ (median: $96.92 \%$ ) (Figures 2E,F).

The mean proportion of $\mathrm{CD}^{+} 6^{+}$mDCs in the patients with AR was $62.15 \pm 7.64 \%$ (median: 64.69\%) and was higher $(p=0.006)$ than the control group, where the mean percentage of these cells was $92.3 \pm 5.84 \%$ (median: 95.12\%) (Figures 2G,H).

\section{Identification of DE IncRNAs and mRNAs in imDCs}

In total, $308 \mathrm{DE}$ mRNAs, including 175 upregulated mRNAs and 133 downregulated mRNAs, were found in the imDCs of patients with AR and NP. A clustergram (Figure 3A) and volcano plots (Figure 3C) are used to depict DE mRNAs. Table 1 shows 67 mRNAs with the largest fold changes. The list contains several genes, including HLA-C, MARCO, KIR2DS3, ITGAV, CD36, and IFNB1. Additionally, 168 DE mRNAs, including 77 upregulated mRNAs and 91 downregulated mRNAs, were found in the mDCs of patients with AR. Figures $4 A, C$ show the clustergram and volcano plots of the DE mRNAs, respectively. Table 2 shows the 10 mRNAs with the greatest variation, and several genes, such as HLA-B, F11R, HLA-DQB1b, HLA-DQB1, and PTAFR, are also shown.

In total, $962 \mathrm{DE}$ lncRNAs, including 434 upregulated and 528 downregulated lncRNAs, were found in patients with AR and NP. The clustergram (Figure 3B) and volcano plots (Figure 3D) show DE lncRNAs. In total, 601 lncRNAs, including 200 upregulated and 401 downregulated lncRNAs, were found in the DE mDCs of the patients with AR. The clustergram in Figure 4B and the volcano plots in Figure 4D show the DE lncRNAs. Tables 1, 3 show the top 10 lncRNAs of imDCs and mDCs with the largest fold changes. The pathways, including interferongamma-mediated signaling pathway, membrane repolarization, and peptide antigen binding, that contribute to the phagocytosis function in imDCs and antigen-presenting function of $\mathrm{mDCs}$ were also identified.

\section{Interaction, Co-expression Network Analysis of DE mRNAs in Patients With AR}

Figure $5 \mathrm{~A}$ shows the interactions of proteins that were coded by DE mRNAs in imDCs. Additionally, Figure 5B shows the coexpression network between DE lncRNAs and mRNAs. TRIM69 has the maximum target mRNAs including 58 DE mRNAs, and SIDT2 has the maximum co-expressed lncRNAs. Table 2 shows the top 10 co-expression pairs in imDCs. lncRNAs exert their biological function as ceRNAs (Wilfried et al., 2016).

We identified 268 target genes after analyzing the possible DE lncRNAs target genes in imDCs. Figure 5C shows the target genes with a combined score of more than 0.9. HLA-C was the target gene of AC108142.1-005, and CD36 was the target gene of FR264384. Moreover, IFNB1 was the target gene of MIR3150B210. The Venn diagram analysis showed that 95 mRNAs were coincided between the 166 DE mRNAs and the 95 DE IncRNA target genes (Figure 5D). The $95 \mathrm{DE}$ lncRNAs were all included in the 166 DE mRNAs.

Figure 6A shows the interaction proteins that were coded by DE mRNAs in mDCs. In this network, HLA-B, HLA-DQB1, HLA-DQB2, PTAFR, and F11R are important genes that interact with many other DE mRNAs. Furthermore, Figure 6B shows the co-expression network of DE lncRNAs and mRNAs. TRIM77P has the maximum target numbers including $39 \mathrm{DE}$ mRNAs, in 

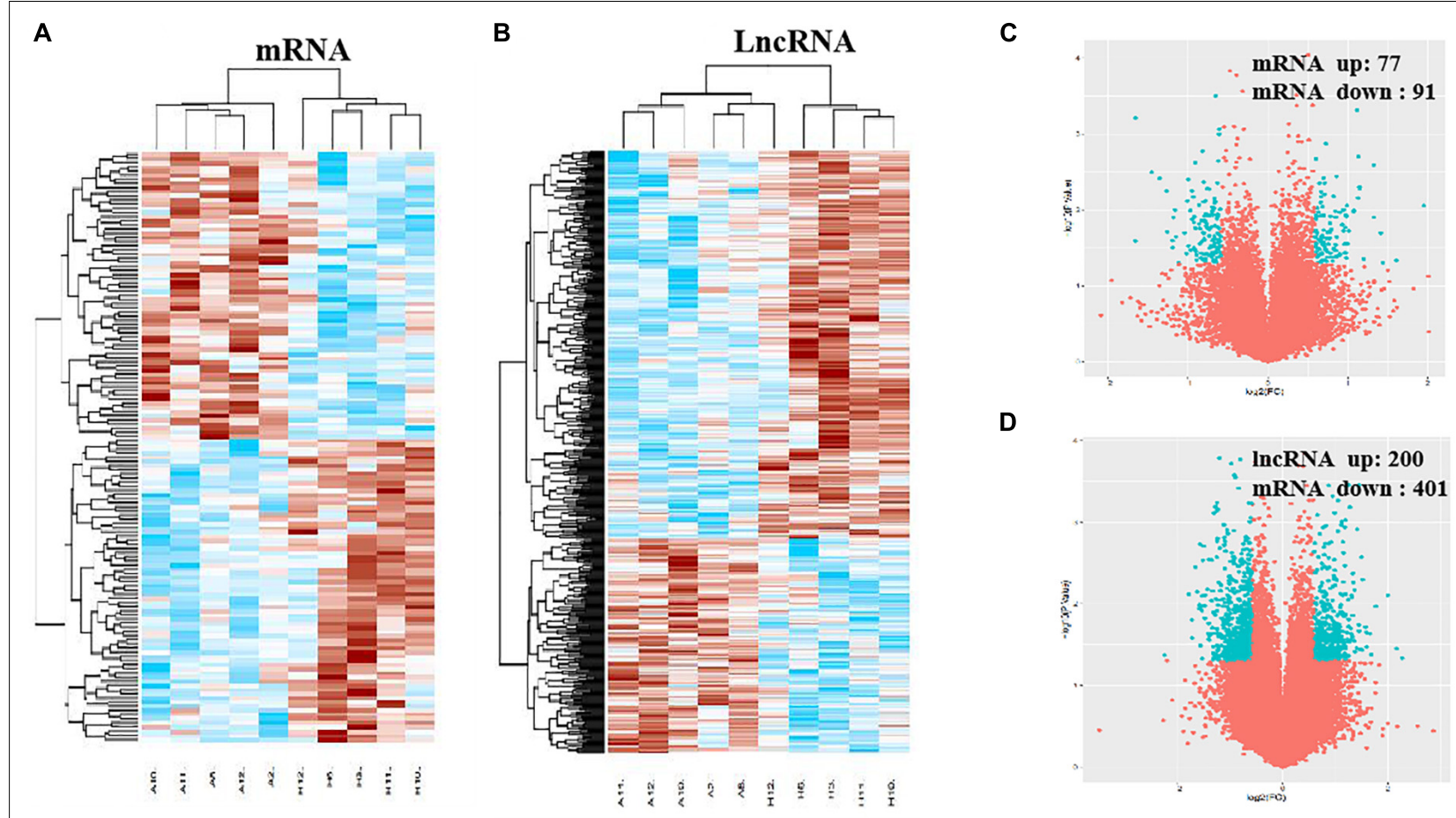

D

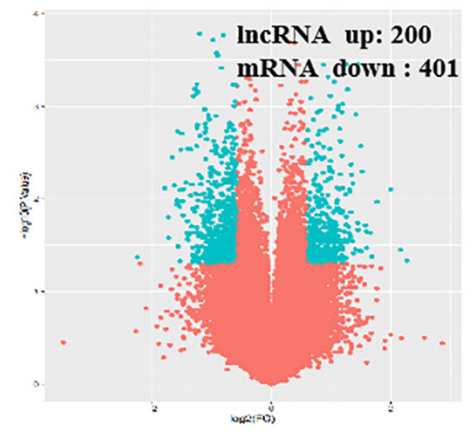

E
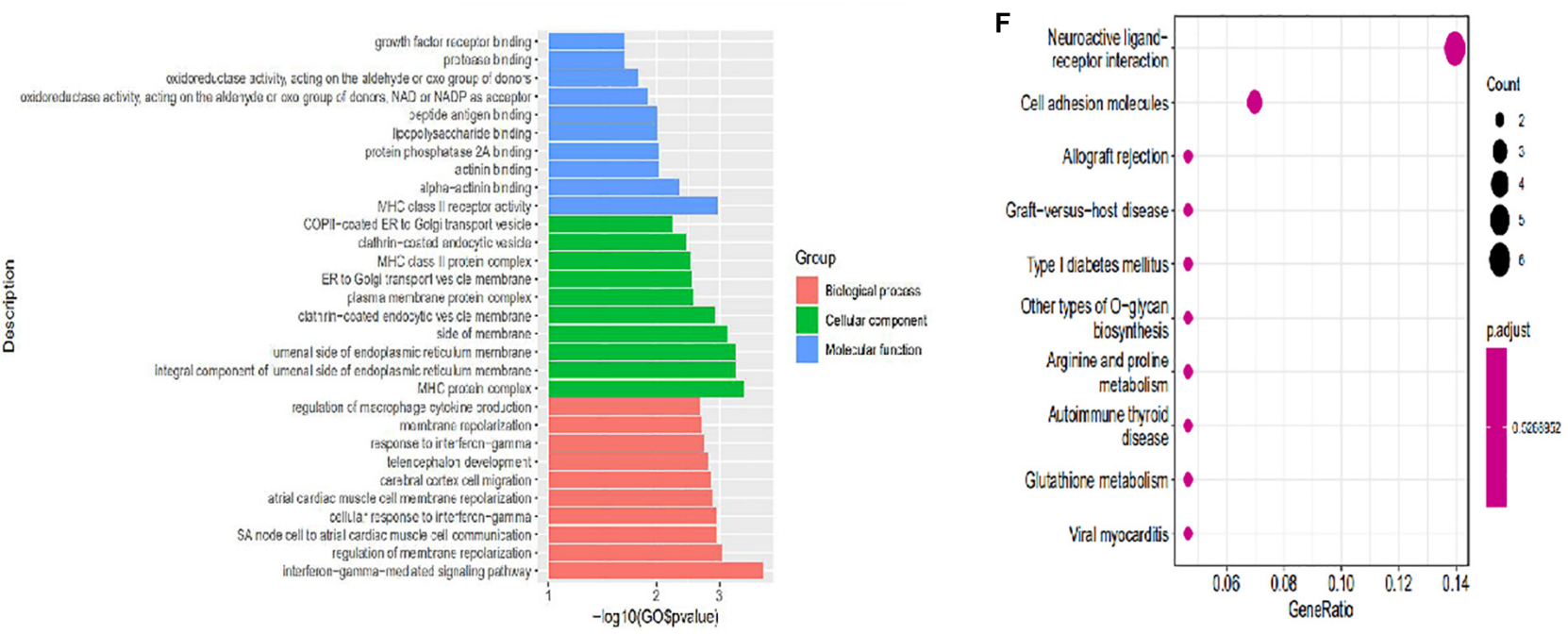

FIGURE 4 | Identification of DE IncRNAs and mRNAs, GO, and KEGG analysis in mDCs. (A) Heatmaps of DE mRNAs between the AR group and NP group. (B) Heatmaps of DE IncRNAs between the AR group and NP group. (C) Volcano plots of DE mRNAs between the AR group and NP group. (D) Volcano plots of DE IncRNAs between the AR group and NP group. (E) Top 10 terms from a GO analysis of molecular function, biological process, and cellular component. (F) DE mRNAs were clustered by KEGG analysis, and the top 10 pathways are shown.

which FAM153A and ZNF396 have the maximum co-expressed lncRNAs. Table 4 shows the top 10 co-expression pairs of mDCs. Additionally, we also investigated the possible presence of $\mathrm{DE}$ lncRNA target genes in mDCs. In our study, 99 target genes were identified in these DE lncRNAs. Figure 6C shows the target genes with the combined score of more than 0.9. In the figure, HLA$\mathrm{B}$ has three target genes of lincRNAs, namely, DHRS3, FCHO2, and linc-PRR5-1. Figure 6D shows that 42 mRNAs coincided between the 95 DE mRNAs and the 42 DE lncRNA target genes in the Venn diagram analysis. Moreover, the $42 \mathrm{DE}$ IncRNAs were included in the $95 \mathrm{DE}$ mRNAs. It also includes some known inflammatory-related molecules.

\section{Validation of DE mRNA and IncRNA Expression Levels by RT-qPCR}

RT-qPCR was used to evaluate DE mRNAs and lncRNAs to verify our RNA chip results. We randomly detected three lncRNAs and 10 mRNAs. MARCO, KIR2DS3, F11R, HLAB, HLA-C, NON-HSAG046717, and NON-HSAT089067 were 
TABLE 2 | The top 10 co-expression of mRNA and IncRNA in imDCs.

\begin{tabular}{|c|c|c|c|c|c|}
\hline mRNA & Gene & IncRNA & Gene & Correlation coefficient & $P$-value \\
\hline NM_018208 & ETNK2 & NON-HSAT008926 & ETNK2 & 0.9970621 & 3.24775E-10 \\
\hline NM_005891 & ACAT2 & NON-HSAG045301 & NON-HSAG045301 & 0.9953173 & 2.09182E-09 \\
\hline NM_182985 & TRIM69 & NR_104175.1 & LOC400799 & -0.9914054 & 2.36259E-08 \\
\hline BX538082 & GPR17 & FR351114 & FR351114 & 0.9906462 & 3.31169E-08 \\
\hline NM_002977 & SCN9A & NON-HSAG029733 & NON-HSAG029733 & 0.9882097 & 8.33527E-08 \\
\hline NM_014485 & HPGDS & NON-HSAT097445 & HPGDS & 0.9877657 & 9.6583E-08 \\
\hline AK131565 & LOC100132368 & ENST00000588609 & LINC00906-004 & 0.9863838 & 1.47941E-07 \\
\hline NM_005005 & NDUFB9 & NON-HSAT101913 & RP11-1113N2.4 & 0.9860654 & 1.62207E-07 \\
\hline XM_001719518 & LOC100128869 & NON-HSAG042749 & NON-HSAG042749 & -0.985726 & 1.78529E-07 \\
\hline NM_032772 & ZNF503 & ENST00000438293 & RP11-88H9.2-003 & 0.9853318 & 1.98986E-07 \\
\hline
\end{tabular}

TABLE 3 | The characteristics of mRNAs with the largest fold change in mDCs.

\begin{tabular}{|c|c|c|c|}
\hline GeneName & Accession no. & Fold change & Regulation \\
\hline Armadillo repeat containing 9 & NM_025139 & 2.1053998 & Up \\
\hline Cat eye syndrome chromosome region, candidate 2 & NM_031413 & 2.1667209 & Down \\
\hline Semenogelin II & NM_003008 & 2.2934558 & Up \\
\hline Polycystic kidney disease 1 like 1 & NM_138295 & 3.8540776 & Up \\
\hline Rho guanine nucleotide exchange factor (GEF) 35 & NM_001003702 & 2.662969 & Up \\
\hline Sushi domain containing 4 & NM_017982 & 3.1600573 & Down \\
\hline ST6 beta-galactosamide alpha-2,6-sialyltranferase 1 & NM_173216 & 2.504244 & UP \\
\hline Chromosome 2 open reading frame 71 & NM_001029883 & 2.1819167 & Up \\
\hline Dehydrogenase/reductase (SDR family) member 3 & NM_004753 & 2.2156718 & Up \\
\hline Sterile alpha motif and leucine zipper containing kinase AZK & NM_016653 & 2.2057335 & UP \\
\hline Chromosome 5 open reading frame 62 & NM_032947 & 2.0991595 & Up \\
\hline Transmembrane protein ENSP00000343375 & BC031304 & 2.0236611 & Down \\
\hline Family with sequence similarity 101, member B & NM_182705 & 2.506473 & Up \\
\hline Olfactory receptor, family 2 , subfamily $T$, member 8 & NM_001005522 & 2.5608928 & Down \\
\hline Sorbin and $\mathrm{SH} 3$ domain containing 1 & NM_001034954 & 2.0617623 & Down \\
\hline Transmembrane protease, serine 6 & BC039082 & 2.2889757 & Down \\
\hline DEAD (Asp-Glu-Ala-Asp) box polypeptide 6 & NM_004397 & 2.0167336 & Up \\
\hline spondin 2, extracellular matrix protein & NM_012445 & 2.7114112 & Up \\
\hline HLA-DBQ1 & NM_001243961 & 2.154 & Down \\
\hline
\end{tabular}

upregulated, whereas ITGAV, CD36, IFNB1, PTAFR, HLADQB1, NON-HSAT 059748, NON-HSAT024276, and NONHSAT098958 were downregulated. The results of RT-qPCR were consistent with those of RNA chip results, hence confirming that our chip data were reliable (Figure 7).

\section{DISCUSSION}

In our research, we used rhGM-CSF, rhIL-4, and TNF- $\alpha$ to induce the mDCs from PBMCs. The generation of human monocyte-derived DCs from whole blood was recognized by all the scientists (Wilfried et al., 2016). We investigated phenotypic and functional features of DE DCs in vitro from patients with AR and the NPs. Besides that, we used RTqPCR to confirm these findings. Our KEGG pathway analysis (Tables 5,6) indicates that interferon-gamma-mediated signaling pathway, membrane repolarization, and peptide antigen binding pathways contribute to the phagocytosis function in imDCs, and the antigen-presenting function in $\mathrm{mDCs}$ contributes to the immunoregulatory function of DCs in AR.

Dendritic cells play a central role in allergic inflammation (Froidure et al., 2016). The latest in vitro techniques allow the in vitro differentiation of DCs (Thurner et al., 1999). Their ability to induce the proliferation of T cells in the MLR assay is commonly used for evaluating their functions (Cao et al., 2004). In MLR experiment, the stimulation index of mDCs was significantly higher than that of the imDCs. In our study, the expression of HLA-DR, CD80, and CD86 in patients with AR is indeed upregulated than that in NP, which is a sign of improved $\mathrm{mDCs}$ antigen presentation in patients with AR. This result is in accordance with a previous study by KleinJan et al. (2006). In the study, CD14 in the NP group was higher than that in patients with AR. CD14 is the marker of monocytes whose expression decreases gradually during DC differentiation from monocytes. In fact, CD80 and CD86 are important co-stimulating factors that affect the proliferation of $\mathrm{T}$ lymphocytes in the $\mathrm{DCs}$ (Duperrier et al., 2000; Ebner et al., 2001; Andreia et al., 2005; 


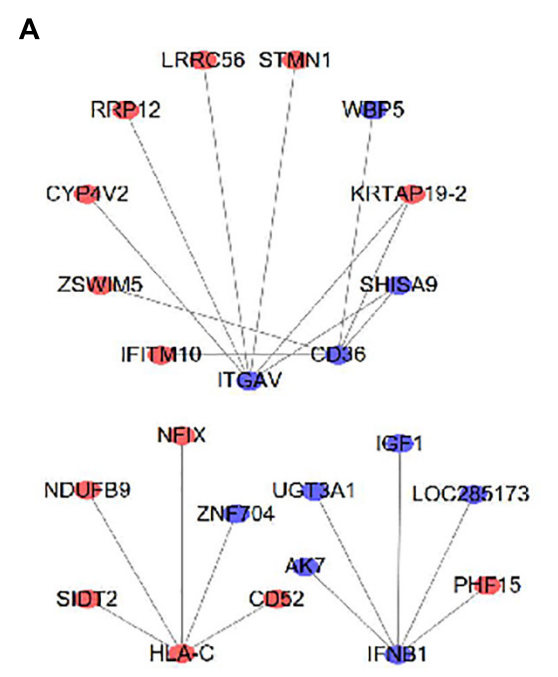

C

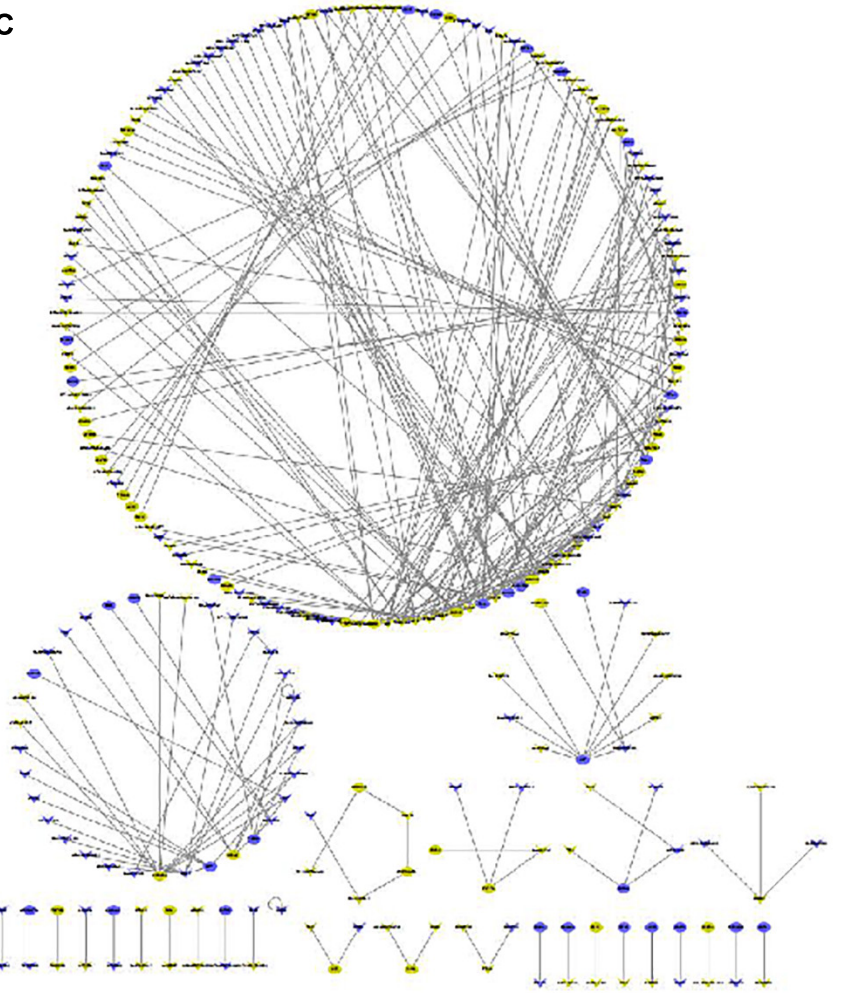

B

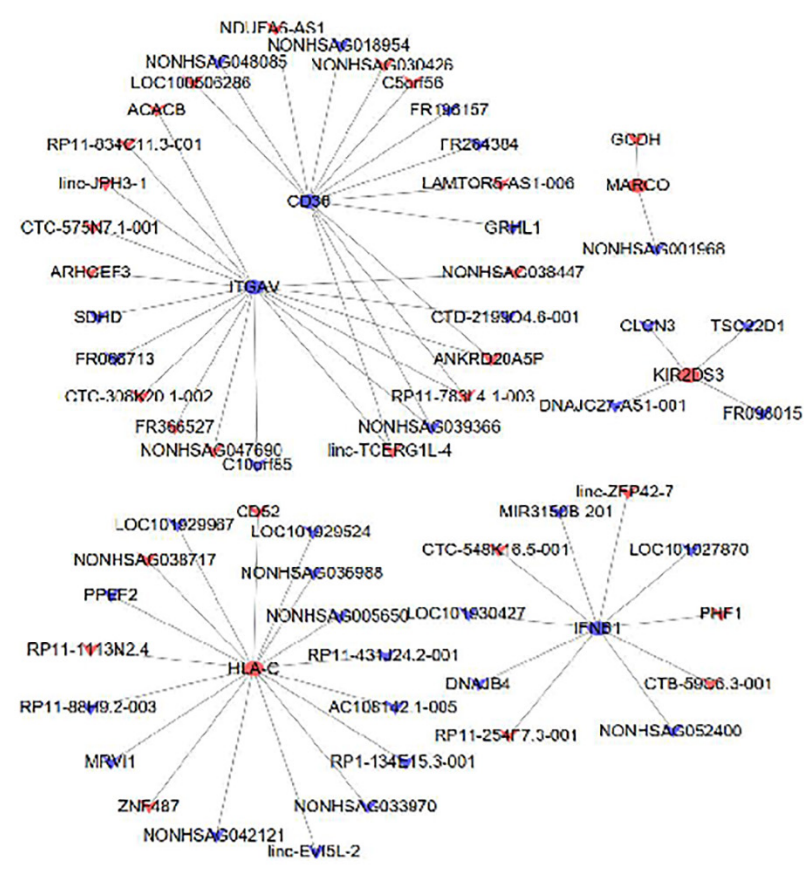

D

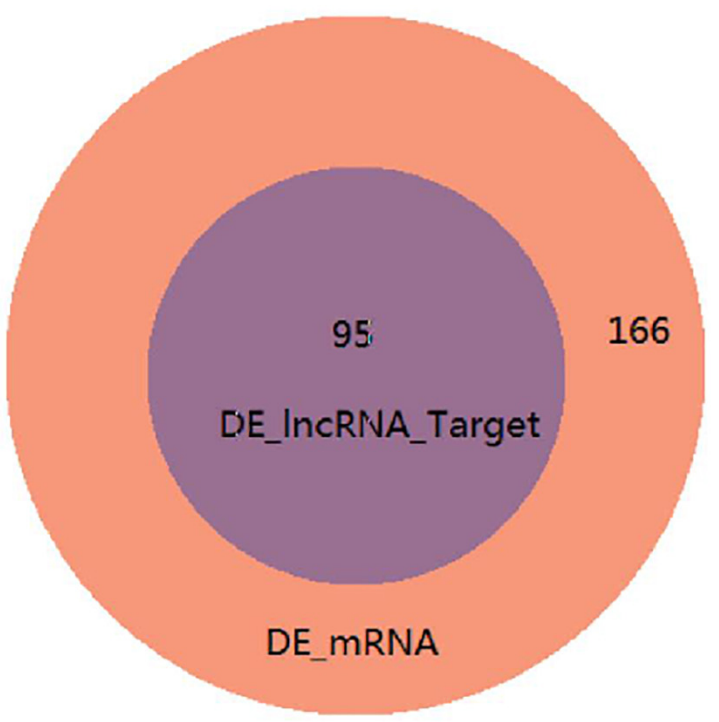

FIGURE 5 | Interaction and co-expression network analysis, analysis of IncRNA target mRNAs in imDCs. (A) Interactions between DE mRNAs. Purple indicates upregulated genes, and green indicates downregulated genes. (B) Co-expression network of DE IncRNAs and DE mRNAs. (C) DE IncRNAs and their target genes with a combined score larger than 0.9 are shown. Yellow indicates upregulated genes, and blue indicates downregulated genes. (D) Venn diagram showing DE mRNAs and target genes of DE IncRNAs.

Wilfried et al., 2016). DCs are the most efficient APCs. It can present the antigens to the $\mathrm{T}$ cells for stimulating the adaptive immune response.
More number of studies have focused on exploring the mRNA expressed in DCs, but none have been conducted to reveal which and how mRNA affects DCs in patients with AR. To investigate 


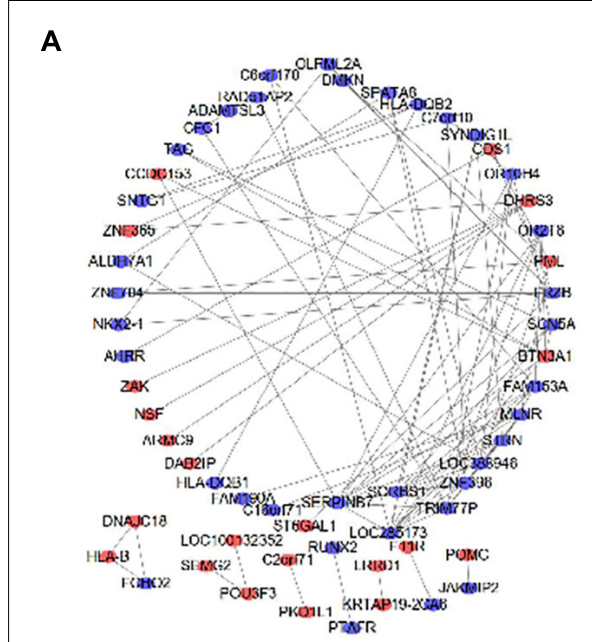

B

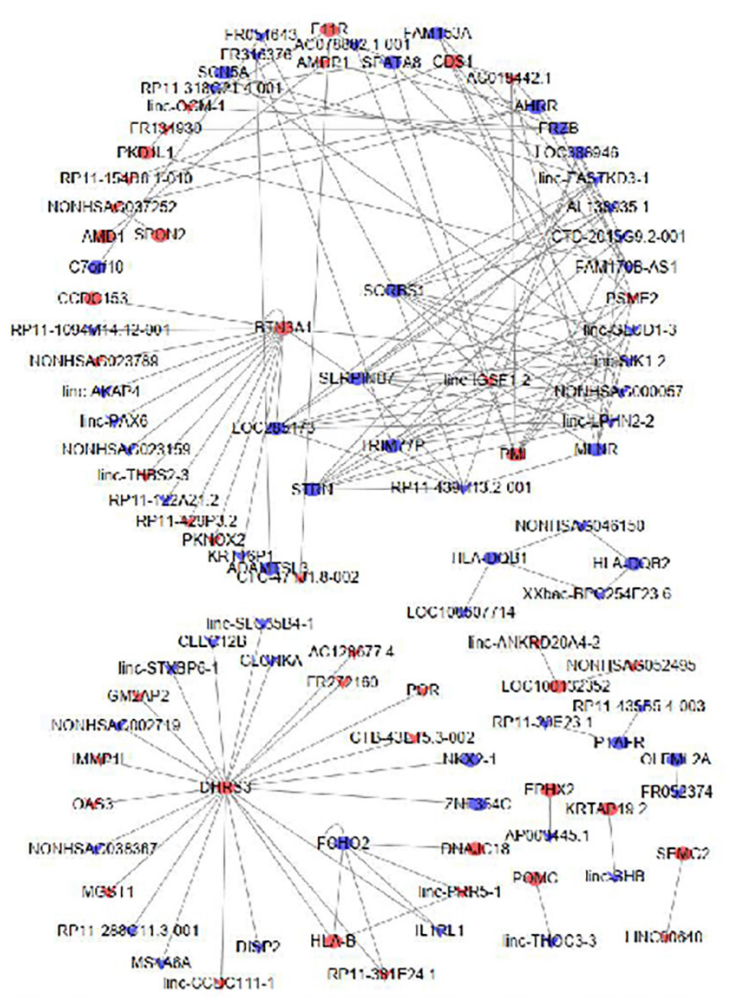

C

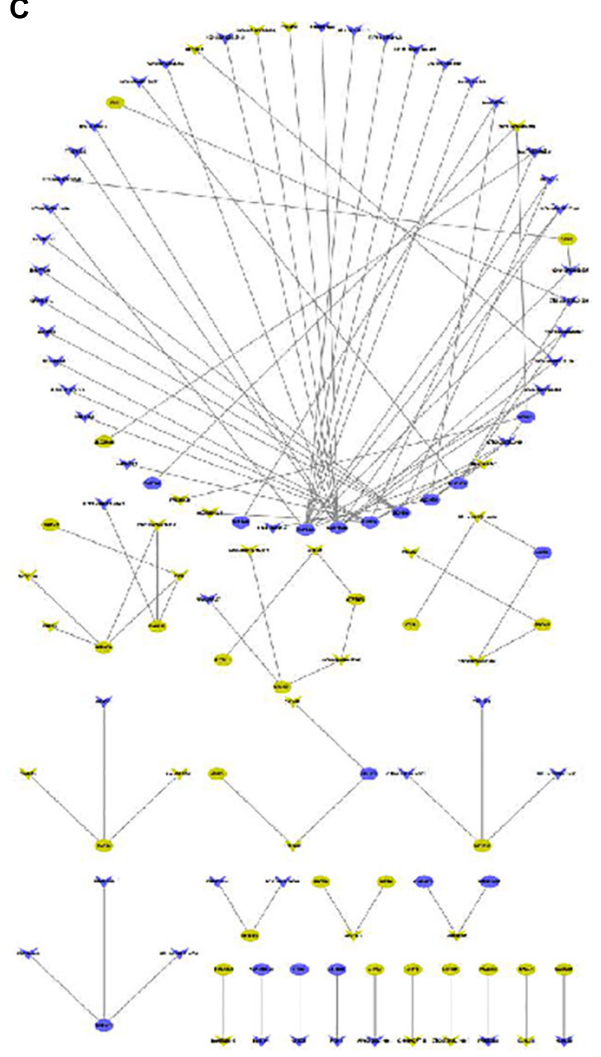

D

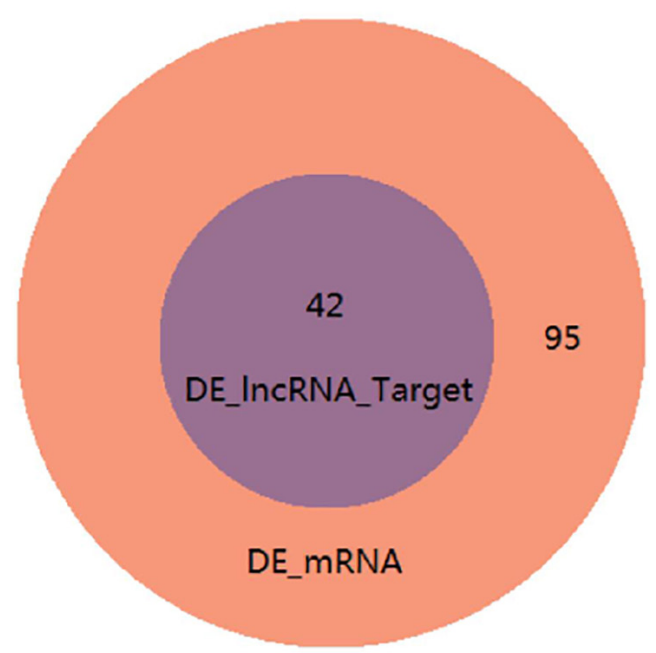

FIGURE 6 | Interaction and co-expression network analysis, analysis of IncRNA target mRNAsin mDCs. (A) Interactions between DE mRNAs. Purple indicates upregulated genes, and green indicates downregulated genes. (B) Co-expression network of DE IncRNAs and DE mRNAs. (C) DE IncRNAs and their target genes with a combined score larger than 0.9 are shown. Yellow indicates upregulated genes, and blue indicates downregulated genes. (D) Venn diagram showing DE mRNAs and target genes of DE IncRNAs.

the mechanism of DCs' functions in patients with AR, the mRNA expression profile was determined and bioinformatics analysis was performed in our study. In total, 308 mRNAs were identified in the analysis. Among these DE genes, HLA-C, ITGAV, MARCO, CD36, IFNB1, and KIR2DS3 were found to be most significantly upregulated in the imDCs' network.
HLA-C plays an important role in promoting differential DC maturation (Raj et al., 2011). ITGAV is the expression of DC-specific transmembrane protein. MARCO promotes TLR activation, which validates a major role of MARCO in mounting an inflammatory response (Haydn et al., 2014). ImDCs play an important role in the phagocytosis of apoptotic 
TABLE 4 | The top 10 co-expression of mRNA and IncRNA in mDCs.

\begin{tabular}{lcccr}
\hline mRNA & Gene & IncRNA & Gene & Correlation coefficient \\
\hline NM_012445 & SPON2 & NON-HSAG037252 & NON-HSAG037252 & 0.9947499 \\
NM_138782 & FCHO2 & NON-HSAT102095 & FCHO2 & $3.30 E-09$ \\
AK311167 & LOC100132352 & TCONS_12_00028804 & linc-ANKRD20A4-2 & 0.9945368 \\
NM_003162 & STRN & ENST00000562064 & CTD-2015G9.2-001 & 0.9832951 \\
NM_001034954 & SORBS1 & TCONS_00010265 & linc-FASTKD3-1 & 0.9825929 \\
NM_001463 & FRZB & NR_038973.1 & FAM170B-AS1 & 0.9794563 \\
NM_001979 & EPHX2 & NON-HSAT021480 & AP000445.1 & 0.9777894 \\
AB018295 & FAM153A & TCONS_00002078 & linc-LPHN2-2 & 3.94 E-07 \\
NM_007048 & BTN3A1 & NON-HSAT142393 & RP11-429P3.2 & 7.60 E-07 \\
NM_182487 & OLFML2A & FR052374 & FR052374 & $1.04 \mathrm{E}-06$ \\
NM_001146162 & TRIM77P & ENST00000541885 & RP11-439H13.2-001 & 1.19 E-06 \\
& & & 0.9747958 & 0.9737392 \\
\end{tabular}
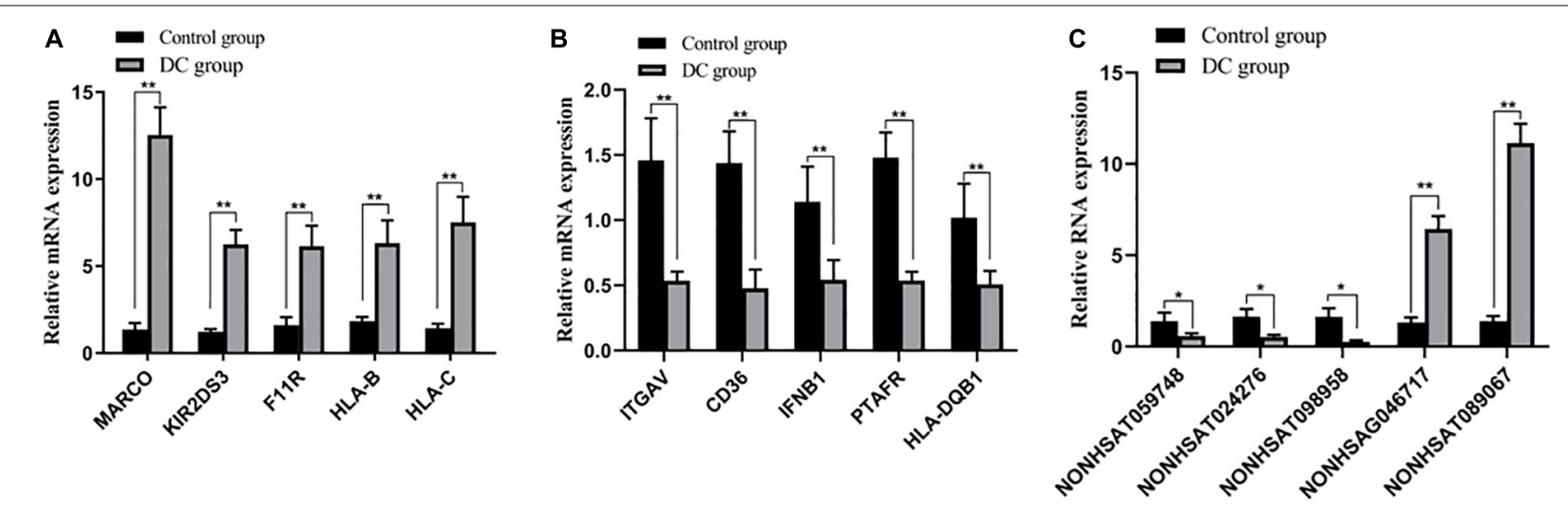

FIGURE 7 | Validation of DE mRNAs and IncRNAs. (A,B) DE mRNAs were confirmed by qPCR. (C) DE IncRNAs were confirmed by qPCR. Ligand 1, MACO, the scavenger receptor; Ligand 2, KIR2DS3, a type of killer cell immunoglobulin-like receptors (KIRs); Ligand 3, F11R, a cell adhesion molecule found on the surface of human platelets; Ligand 4 and Ligand 5, HLA-B and HLA-C are all a type of human Leukocyte Antigen (HLA) class I molecules; Ligand 6, ITGAV, integrin subunit alpha v; Ligand 7, CD36, a membrane glycoprotein present on platelets, mononuclear phagocytes, adipocytes, hepatocytes, myocytes, and some epithelia; Ligand 8, IFNB1, interferon beta, a secreted cytokine; Ligand 9, PTAFR, platelet-activating factor receptor; Ligand 10, HLA-DQB1, human leukocyte antigen DQB1. ${ }^{\star *} P<0.01,{ }^{*} P<0.05$

TABLE 5 | Pathways KEGG analysis of imDCs.

\begin{tabular}{lcccc}
\hline Pathway & Count & $\boldsymbol{P}$-value & Corrected $\boldsymbol{P}$-value & Gene \\
\hline Vitamin digestion and absorption & 3 & 0.003377492 & 0.355867536 & PLB1/SLC19A1/SCARB1 \\
Butanoate metabolism & 3 & 0.005268382 & 0.355867536 & AACS/ACADS/ACAT2 \\
Hypertrophic cardiomyopathy & 5 & 0.005764726 & 0.355867536 & TPM2/TPM1/MYH7/ITGAV/IGF1 \\
Dilated cardiomyopathy & 5 & 0.00755082 & 0.355867536 & TPM2/TPM1/MYH7/ITGAV/IGF1 \\
Aldosterone synthesis and secretion & 5 & 0.008223347 & 0.355867536 & CACNA1//KCNJ5/PRKCB/SCARB1/POMC \\
GnRH secretion & 4 & 0.008896688 & 0.355867536 & CACNA11/KCNJ5/PRKCB/PIK3R3 \\
Aldosterone-regulated sodium reabsorption & 3 & 0.011502085 & 0.390782867 & PRKCB/PIK3R3/IGF1 \\
Phagosome & 6 & 0.013026096 & 0.390782867 & C1R/ITGAV/SCARB1/MARCO/CD36/HLA-C \\
Glioma & 4 & 0.015303573 & 0.408095288 & PRKCB/PIK3R3/CDK6/IGF1 \\
Fat digestion and absorption & 3 & 0.017291278 & 0.414990664 & SCARB1/CD36/ACAT2 \\
Valine, leucine, and isoleucine degradation & 3 & 0.023152058 & 0.479306938 & AACS/ACADS/ACAT2 \\
Cholesterol metabolism & 3 & 0.025762258 & 0.479306938 & LRPAP1/SCARB1/CD36 \\
Natural killer cell-mediated cytotoxicity & 5 & 0.025962459 & 0.479306938 & IFNB1/PRKCB/PIK3R3/KIR2DS3/HLA-C \\
Terpenoid backbone biosynthesis & 2 & 0.031653591 & 0.542632982 & MVD/ACAT2 \\
Inflammatory mediator regulation of TRP channels & 4 & 0.038848883 & 0.574014469 & PRKCB/PIK3R3/IGF1/P2RY2 \\
Parathyroid hormone synthesis, secretion, and action & 4 & 0.046482901 & 0.574014469 & MMP14/RUNX2/PRKCB/PDE4D
\end{tabular}


TABLE 6 | Pathways of mDCs with the largest significant difference in KEGG analysis.

\begin{tabular}{|c|c|c|c|c|}
\hline Pathway & Count & $P$-value & Corrected $P$-value & Gene \\
\hline Neuroactive ligand-receptor interaction & 6 & 0.008916046 & 0.526895235 & PTAFR, POMC, OPRM1, MCHR2, GNRH1, MLNR \\
\hline Allograft rejection & 2 & 0.017316097 & 0.526895235 & HLA-DQB1, HLA-B \\
\hline Graft-versus-host disease & 2 & 0.020925187 & 0.526895235 & HLA-DQB1, HLA-B \\
\hline Type I diabetes mellitus & 2 & 0.021872484 & 0.526895235 & HLA-DQB1, HLA-B \\
\hline Other types of O-glycan biosynthesis & 2 & 0.025835563 & 0.526895235 & ST6GAL1, LFNG \\
\hline Arginine and proline metabolism & 2 & 0.028984793 & 0.526895235 & AMD1, ALDH7A1 \\
\hline Autoimmune thyroid disease & 2 & 0.032279732 & 0.526895235 & HLA-DQB1, HLA-B \\
\hline Glutathione metabolism & 2 & 0.036890658 & 0.526895235 & GSTM3, G6PD \\
\hline Viral myocarditis & 2 & 0.04050554 & 0.526895235 & HLA-DQB1, HLA-B \\
\hline Cell adhesion molecules & 3 & 0.044652139 & 0.526895235 & HLA-DQB1, HLA-B, F11R \\
\hline Retinol metabolism & 2 & 0.050761992 & 0.544537736 & DHRS3, ALDH1A1 \\
\hline
\end{tabular}

TABLE 7 | GO analysis of DE mRNA in imDCs.

\begin{tabular}{|c|c|c|c|c|}
\hline Term & Domain & Count & $P$-value & Corrected $\boldsymbol{P}$-value \\
\hline Apoptotic cell clearance & Biological process & 5 & 4.46014E-05 & 4.46014E-05 \\
\hline Vasculogenesis & Biological process & 6 & 0.000164507 & 0.000164507 \\
\hline Response to fatty acid & Biological process & 6 & 0.000263738 & 0.000263738 \\
\hline Phospholipid metabolic process & Biological process & 13 & 0.000319148 & 0.000319148 \\
\hline Glycerophospholipid metabolic process & Biological process & 11 & 0.000323952 & 0.000323952 \\
\hline Oligodendrocyte differentiation & Biological process & 6 & 0.000454451 & 0.000454451 \\
\hline Actin filament-based movement & Biological process & 7 & 0.00047494 & 0.00047494 \\
\hline Glycerolipid metabolic process & Biological process & 12 & 0.000748476 & 0.000748476 \\
\hline Negative regulation of tumor necrosis factor production & Biological process & 5 & 0.000769209 & 0.000769209 \\
\hline Carbohydrate derivative transport & Biological process & 5 & 0.000769209 & 0.000769209 \\
\hline Synaptic vesicle membrane & Cellular component & 5 & 0.002649207 & 0.002649207 \\
\hline Exocytic vesicle membrane & Cellular component & 5 & 0.002649207 & 0.002649207 \\
\hline Integral component of synaptic vesicle membrane & Cellular component & 3 & 0.003805305 & 0.003805305 \\
\hline Presynaptic cytosol & Cellular component & 2 & 0.008553871 & 0.008553871 \\
\hline Presynapse & Cellular component & 11 & 0.008923316 & 0.008923316 \\
\hline Cell leading edge & Cellular component & 10 & 0.009798418 & 0.009798418 \\
\hline Integral component of organelle membrane & Cellular component & 6 & 0.009895744 & 0.009895744 \\
\hline Exocytic vesicle & Cellular component & 6 & 0.009895744 & 0.009895744 \\
\hline Melanosome membrane & Cellular component & 2 & 0.00990825 & 0.00990825 \\
\hline Chitosome & Cellular component & 2 & 0.00990825 & 0.00990825 \\
\hline Transforming growth factor beta binding & Molecular function & 4 & 7.69207E-05 & 7.69207E-05 \\
\hline 1-Phosphatidylinositol binding & Molecular function & 3 & 0.000452198 & 0.000452198 \\
\hline Lipoprotein particle receptor activity & Molecular function & 3 & 0.000452198 & 0.000452198 \\
\hline Signaling pattern recognition receptor activity & Molecular function & 3 & 0.000824225 & 0.000824225 \\
\hline Pattern recognition receptor activity & Molecular function & 3 & 0.000980996 & 0.000980996 \\
\hline Amyloid-beta binding & Molecular function & 5 & 0.001167764 & 0.001167764 \\
\hline Lipase activity & Molecular function & 5 & 0.005214971 & 0.005214971 \\
\hline Intronic transcription regulatory region sequence-specific DNA binding & Molecular function & 2 & 0.005236034 & 0.005236034 \\
\hline Intronic transcription regulatory region DNA binding & Molecular function & 2 & 0.005236034 & 0.005236034 \\
\hline Lipoprotein particle binding & Molecular function & 3 & 0.006313499 & 0.006313499 \\
\hline
\end{tabular}

cells, in particular, CD36 (Albert et al., 1998). Additionally, the GO analysis demonstrated specific molecular functions, for example, binding of transforming growth factor-beta, signaling pattern recognition receptor activity, and pattern recognition receptor activity, thereby indicating the critical role of these cytokines in the immunoregulatory functions of imDCs. The KEGG analysis identified 17 signaling pathways of the DE mRNAs, wherein interferon-gamma-mediated signaling pathway, membrane repolarization, and peptide antigen binding were the pathways with the significant differences that contributed to the phagocytosis function of imDCs (Figures 3E,F). This result is consistent with that of a previous study (Lambrecht, 2001). Although research works have been conducted to determine the functions of imDCs in AR, the 
TABLE 8 | GO analysis of DE mRNA in mDCs.

\begin{tabular}{|c|c|c|c|c|}
\hline Term & Domain & Count & $P$-value & Corrected $P$-value \\
\hline Interferon-gamma-mediated signaling pathway & Biological process & 5 & 0.000105145 & 0.000105145 \\
\hline Regulation of membrane repolarization & Biological process & 3 & 0.000897665 & 0.000897665 \\
\hline SA node cell to atrial cardiac muscle cell communication & Biological process & 2 & 0.001143106 & 0.001143106 \\
\hline Cellular response to interferon-gamma & Biological process & 5 & 0.001152483 & 0.001152483 \\
\hline Atrial cardiac muscle cell membrane repolarization & Biological process & 2 & 0.001392464 & 0.001392464 \\
\hline Cerebral cortex cell migration & Biological process & 3 & 0.001490903 & 0.001490903 \\
\hline Telencephalon development & Biological process & 6 & 0.001681684 & 0.001681684 \\
\hline Response to interferon-gamma & Biological process & 5 & 0.001981297 & 0.001981297 \\
\hline Membrane repolarization & Biological process & 3 & 0.002157971 & 0.002157971 \\
\hline Regulation of macrophage cytokine production & Biological process & 2 & 0.002280906 & 0.002280906 \\
\hline MHC protein complex & Cellular component & 3 & 0.000314384 & 0.000314384 \\
\hline Integral component of lumenal side of endoplasmic reticulum membrane & Cellular component & 3 & 0.000491764 & 0.000491764 \\
\hline Lumenal side of endoplasmic reticulum membrane & Cellular component & 3 & 0.000491764 & 0.000491764 \\
\hline Side of membrane & Cellular component & 8 & 0.000689806 & 0.000689806 \\
\hline Clathrin-coated endocytic vesicle membrane & Cellular component & 3 & 0.001274169 & 0.001274169 \\
\hline Plasma membrane protein complex & Cellular component & 8 & 0.002912282 & 0.002912282 \\
\hline ER to Golgi transport vesicle membrane & Cellular component & 3 & 0.003192827 & 0.003192827 \\
\hline MHC class II receptor activity & Cellular component & 2 & 0.003242854 & 0.003242854 \\
\hline Alpha-actinin binding & Cellular component & 3 & 0.003895823 & 0.003895823 \\
\hline Actinin binding & Cellular component & 3 & 0.006041689 & 0.006041689 \\
\hline Protein phosphatase 2A binding & Molecular function & 3 & 0.000314384 & 0.000314384 \\
\hline Lipopolysaccharide binding & Molecular function & 3 & 0.000491764 & 0.000491764 \\
\hline Peptide antigen binding & Molecular function & 3 & 0.000491764 & 0.000491764 \\
\hline $\begin{array}{l}\text { Oxidoreductase activity, acting on the aldehyde or oxo group of donors, NAD or } \\
\text { NADP as acceptor }\end{array}$ & Molecular function & 8 & 0.000689806 & 0.000689806 \\
\hline Oxidoreductase activity, acting on the aldehyde or oxo group of donors & Molecular function & 3 & 0.001274169 & 0.001274169 \\
\hline Protease binding & Molecular function & 8 & 0.002912282 & 0.002912282 \\
\hline Growth factor receptor binding & Molecular function & 3 & 0.003192827 & 0.003192827 \\
\hline MHC class II receptor activity & Molecular function & 2 & 0.003242854 & 0.003242854 \\
\hline Alpha-actinin binding & Molecular function & 3 & 0.003895823 & 0.003895823 \\
\hline Actinin binding & Molecular function & 3 & 0.006041689 & 0.006041689 \\
\hline
\end{tabular}

TABLE 9 | IncRNAs in imDCs with the largest fold change.

\begin{tabular}{|c|c|c|c|c|c|c|c|c|}
\hline IncRNA_Accession & FC (abs) & Regulation & Chromosome & Strand & Start & End & Class & Size \\
\hline TCONS_I2_00030438 & 6.1069527 & Down & $\operatorname{chrX}$ & - & 5571461 & 5644346 & IncRNA & 72885 \\
\hline NON-HSAT016934 & 5.6181483 & Down & $\operatorname{chr10}$ & - & 127823936 & 127843874 & IncRNA & 19938 \\
\hline TCONS_I2_00001274 & 5.147873 & Up & chr1 & - & 65450880 & 65451399 & IncRNA & 519 \\
\hline NON-HSAT016933 & 4.9528494 & Down & chr10 & - & 127779304 & 127798357 & IncRNA & 19053 \\
\hline NON-HSAT059748 & 4.615665 & Down & $\operatorname{chr18}$ & + & 66817065 & 66832387 & IncRNA & 15322 \\
\hline NON-HSAG053933 & 4.34199 & Up & $\operatorname{chrX}$ & - & 2484070 & 2527190 & IncRNA & 43120 \\
\hline NON-HSAG029733 & 4.2480536 & Down & chr2 & - & 167054881 & 167055243 & IncRNA & 362 \\
\hline NON-HSAT093933 & 4.0003886 & Down & chr3 & + & 188985384 & 189038493 & IncRNA & 53109 \\
\hline NON-HSAG036957 & 3.8607297 & Down & chr3 & + & 188985385 & 189038493 & IncRNA & 53108 \\
\hline NON-HSAG055855 & 3.8563106 & Down & chrY & - & 21034387 & 21239448 & IncRNA & 205061 \\
\hline
\end{tabular}

role of IFNB1 and KIR2DS3 in imDCs was not known in AR (Tables 7, 8).

The imDCs migrate to the lymphoid organs that will be matured in the future. They present captured Ag to the naïve $\mathrm{T}$ cells (Banchereau and Steinman, 1998). Hence, the imDCs and $\mathrm{mDCs}$ had different functions in the presented Ag. In our study, we focused on the immature and mature stages of
DCs. In the mDC' mRNA analysis, 168 mRNAs were identified. Among these DE genes, HLA-B, HLA-DQB1, HLA-DQB2, PTAFR, and F11R were the most significantly upregulated genes in mDCs. HLA-B is the major histocompatibility complex (class I) antigens that present the processed antigens. HLADQB1 is the major histocompatibility complex (class II) antigens that have been identified as useful biomarkers of 
TABLE 10 | The characteristics of IncRNAs with the largest fold change in mDCs.

\begin{tabular}{|c|c|c|c|c|c|c|c|c|}
\hline IncRNA_Accession & FC (abs) & Regulation & Chromosome & Strand & Start & End & Class & Size \\
\hline NON-HSAT016933 & 4.767738 & Down & chr10 & - & 127779304 & 127798357 & lincRNA & 19053 \\
\hline NON-HSAT005246 & 3.4715514 & Down & chr1 & - & 113068497 & 113084597 & lincRNA & 16100 \\
\hline NR_038346.1 & 3.311445 & Down & chr7 & + & 79082272 & 79100524 & non-coding RNA & 18252 \\
\hline NON-HSAG038966 & 3.027513 & Down & chr4 & - & 141364352 & 141419531 & lincRNA & 55179 \\
\hline NON-HSAG037252 & 3.011491 & Up & chr4 & - & 1165171 & 1202750 & lincRNA & 37579 \\
\hline NON-HSAG008700 & 2.9691923 & Up & chr11 & - & 65556522 & 65562174 & lincRNA & 5652 \\
\hline ENST00000450847 & 2.9003682 & Down & chr1 & - & 248647546 & 248648785 & antisense & 1239 \\
\hline ENST00000541885 & 2.8448925 & Down & chr12 & - & 64900946 & 64927418 & lincRNA & 26472 \\
\hline TCONS_00007688 & 2.8411803 & Up & chr4 & + & 185427281 & 185436808 & lincRNA & 9527 \\
\hline
\end{tabular}

candidacy for effective allergy immunotherapy in patients with AR (Yanming et al., 2019). This result indicates that these cytokines affect the antigen-presenting process in the mDCs. We can determine from the KEGG analysis that there are 12 signaling pathways related to DE mRNAs. The cell adhesion molecules were the useful pathways that contribute to the antigen-presenting function in mDCs (Figures $4 \mathrm{E}, \mathrm{F}$ ), which is consistent with a previous study (Roche and Furuta, 2015). Although many genes have been identified to play an important role in mDCs, but the mechanisms by which HLADQB2, PTAFR, and F11R affect $\mathrm{mDC}$ in $\mathrm{AR}$ are unknown (Tables 9, 10).

Long non-coding RNA is an important component in the mRNA expression profiles (Kopp and Mendell, 2018). Several studies have shown that the differentiation of DCs is closely related to lncRNAs (Pin et al., 2014; Majid et al., 2020). It is confirmed from our studies that lncRNA may play a key role in the development of DC. Our data showed that 172 lncRNAs of imDCs and 104 lncRNAs of mDCs were significantly expressed in patients with $\mathrm{AR}$ as compared to that in NP by at least twofold changes. This result helps in studying the AR-related global transcriptome.

Long non-coding RNAs do not have a protein coding function, but they can be used as a new modulator, such as cis- or transgene regulating expression, demethylation-promoting effect, and mRNA-processing control were the major mechanisms (Sone et al., 2007; Wahlestedt, 2013; Qiao et al., 2016). To analyze the functions of lncRNAs, mRNA-lncRNA was used to create a co-expression profile to predict the potential functions of the DE lncRNAs of patients with AR. We found that the DE lncRNAs were all included in the results of mRNA-lncRNA chip results.

We used RT-qPCR to validate the mRNA-lncRNA chip results with only one randomly selected transcript, and found that the RT-qPCR results are consistent with the chip results in better understanding the role of lncRNA in the pathogenesis of DCmediated AR.

In summary, our study proved that several mRNAs and lncRNAs of DC affect a certain process of AR pathogenesis by regulating the target genes. This result points out the direction for future studies on determining and explaining the functions and mechanisms of AR-related mRNA and lncRNA, and provides new therapeutic targets for patients with AR.

\section{DATA AVAILABILITY STATEMENT}

The original contributions presented in the study are included in the article/Supplementary Material. Further inquiries can be directed to the corresponding author/s.

\section{ETHICS STATEMENT}

The studies involving human participants were reviewed and approved by the medical and experimental animal ethics committee of Beijing University of Traditional Chinese Medicine. The patients/participants provided their written informed consent to participate in this study.

\section{AUTHOR CONTRIBUTIONS}

$\mathrm{YZ}, \mathrm{XC}$, and $\mathrm{YZ}$ carried out the experiments. JW, QW, $\mathrm{YZ}, \mathrm{XC}$, and $\mathrm{YZ}$ designed the study and edited the manuscript. All authors read and approved the final manuscript.

\section{FUNDING}

This work was supported by grants from the National Natural Science Foundation of China (No. 81973715) and Beijing Natural Science Foundation of China (No. 7202110).

\section{SUPPLEMENTARY MATERIAL}

The Supplementary Material for this article can be found online at: https://www.frontiersin.org/articles/10.3389/fcell.2021. 636477/full\#supplementary-material 


\section{REFERENCES}

Albert, M. L., Pearce, S. F., Francisco, L. M., Sauter, B., Roy, P., Silverstein, R. L., et al. (1998). Immature dendritic cells phagocytose apoptotic cells via alphavbeta5 and CD36, and cross-present antigens to cytotoxic T lymphocytes. J Exp Med. 188, 1359-1368. doi: 10.1084/jem.188.7.1359

Andreia, R. N., Luis, F. C. E., Anselmo, L. B., Leite, K. R. M., Buzaid, A. C., Câmara-Lopes, L. H., et al. (2005). Dendritic cells derived from metastatic cancer patients vaccinated with allogeneic dendritic cell- autologous tumor cell hybrids express more CD86 and induce higher levels of interferon-gamma in mixed lymphocyte reactions. Cancer Immunol. Immunother. 54, 61-66. doi: 10.1007/s00262-004-0550-8

Banchereau, J., and Steinman, R. M. (1998). Dendritic cells and the control of immunity. Nature 392, 245-252. doi: 10.1038/32588

Bousquet, J., Khaltaev, N., CruzA, A., Denburg, J., Fokkens, W. J., Togias, A., et al. (2008). Allergic rhinitis and its impact on asthma (ARIA) 2008 update (in collaboration with the World Health Organization, GA (2) LEN and Aller Gen). Allergy 63, 8-160.

Cao, M.-D., Chen, Z.-D., and Xing, Y. (2004). Gamma irradiation of human dendritic cells influences proliferation and cytokine profile of $\mathrm{T}$ cells in autologous mixed lym- phocyte reaction. Cell Biol. Int. 28, 223-228. doi: 10.1016/j.cellbi.2003.12.006

Chang, C. C., Lin, C. C., Hsieh, W. L., Lai, H. W., Tsai, C. H., and Cheng, Y. W. (2014). MicroRNA expression profiling in PBMCs: a potential diagnostic biomarker of chronic hepatitis C. Dis. Markers 2014:157.

Delves, P. J. (2016). Roitt's essential immunology, 11th edn. Trans. R. Soc. Trop. Med. Hyg. 96, 108-108.

Du, M., Yuan, L., Tan, X., Huang, D., Wang, X., Zheng, Z., et al. (2017). The LPSinducible lncRNA Mirt2 is a negative regulator of inflammation. Nat. Commun. 8:2049.

Duperrier, K., Eljaafari, A., Dezutter-Dambuyant, C., Bardin, C., Jacquet, C., Yoneda, K., et al. (2000). Distinct subsets of dendritic cells resembling dermal DCs can be generated in vitro from monocytes, in the presence of different serum supplements. J. Immunol. Methods 238, 119-131. doi: 10.1016/s00221759(00)00147-2

Ebner, S., Ratzinger, G., Krosbacher, B., Schmuth, M., Weiss, A., Reider, D., et al. (2001). Production of IL-12 by human monocyte-derived dendritic cells is optimal when the stimulus is given at the onset of maturation, and is further enhanced by IL-4. J. Immunol. 166, 633-641. doi: 10.4049/jimmunol.166.1.633

Froidure, A., Shen, C., and Pilette, C. (2016). Dendritic cells revisited in human allergic rhinitis and asthma. Allergy 71, 137-148. doi: 10.1111/all.12770

Haydn, T. K., Dunn, L. K., Ghosh, S., Nechama, M., Kobzik, L., and Arredouani, M. S. (2014). The scavenger receptor MARCO modulates TLR- induced responses in dendritic cells. PLoS One 9:e104148. doi: 10.1371/journal.pone. 0104148

KleinJan, A., Willart, M., vanRijt, L. S., Braunstahl, G. J., Leman, K., Jung, S., et al. (2006). An essential role for dendritic cells in human and expe-rimental allergic rhinitis. J. Allergy Clin. Immunol. 1185, 1117-1125. doi: 10.1016/j.jaci.2006. 05.030

Kopp, F., and Mendell, J. T. (2018). Functional classification and experimental dissection of long noncoding RNAs. Cell 172, 393-407. doi: 10.1016/j.cell.2018. 01.011

Lambrecht, B. N. (2001). Allergen uptake and presentation by dendritic cells. Curr. Opin. Allergy Clin. Immunol. 1, 51-59. doi: 10.1097/01.all.0000010985.57414.74

Leynaert, B., Neukirch, C., Kony, S., Guénégou, A., Bousquet, J., Aubier, M., et al. (2004). Association between asthma and rhinitis according to atopic sensitization in a population-based study. J. Allergy Clin. Immunol. 113, 86-93. doi: 10.1016/j.jaci.2003.10.010

Majid, P. K., Rakhshi, N., Aleagha, M. S. E., Abdari, M., Alikhah, A., Safarian, G., et al. (2020). Differential expression of STAT3 gene and its regulatory long noncoding RNAs, namely lnc-DC and THRIL, in two eastern Iranian ethnicities with multiple sclerosis. Neurol Sci. 41, 561-568. doi: 10.1007/s10072-01904092-y

Ouyang, Y., Miyata, M., Hatsushika, K., Ohnuma, Y., Katoh, R., Ogawa, H., et al. (2010). TGF-beta signaling may play a role in the development of goblet cell hyperplasia in a mouse model of allergic rhinitis. Allergol. Int. 59, 313-319. doi: 10.2332/allergolint.10-sc-0172

Pin, W., Xue, Y., Han, Y., Lin, L., Wu, C., Xu, S., et al. (2014). The STAT3-binding long noncoding RNA lnc-DC controls human dendritic cell differentiation. Science 344, 310-313. doi: 10.1126/science.1251456

Poggi, A., Canevali, P., Contatore, M., and Ciprandi, G. (2012). Higher frequencies of CD161+ circulating $\mathrm{t}$ lymphocytes in allergic rhinitis patients compared to healthy donors. Int. Arch. Allergy Immunol. 158, 151-156. doi: 10.1159/ 000330903

Qiao, J., Yao, H., Xia, Y., Chu, P., Li, M., Wu, Y., et al. (2016). Long non-coding RNAs expression profiles in hepatocytes of mice after hematopoietic stem cell transplantation. IUBMB Life 68, 232-241. doi: 10.1002/iub.1479

Raj, H., Kubal, C., Moore, J., Neil, D., Cook, M., Ball, S., et al. (2011). KIR and HLA-C interactions promote differential dendritic cell maturation and is a major determinant of graft failure following kidney transplantation. PLoS One 6:e23631. doi: 10.1371/journal.pone.0023631

Roche, P. A., and Furuta, K. (2015). The ins and outs of mhc class-II-mediated antigen processing and presentation. Nat. Rev. Immunol. 15, 203-216. doi: $10.1038 /$ nri3818

Schiekofer, S., Andrassy, M., Chen, J., Rudofsky, G., Schneider, J., Wendt, T., et al. (2003). Acute hyperglycemia causes intracellular formation of CML and activation of ras, p42/44 MAPK, and nuclear factor kappaB in PBMCs. Diabetes 52, 621-633. doi: 10.2337/diabetes.52.3.621

Sone, M., Hayashi, T., Tarui, H., Agata, K., Takeichi, M., and Nakagawa, S. (2007). The mRNA-like noncoding RNA Gomafu constitutes a novel nuclear domain in a subset of neurons. J. Cell Sci. 120, 2498-2506. doi: 10.1242/jcs.009357

Tacconi, S., Perri, R., Balestrieri, E., Grelli, S., Bernardini, S., Annichiarico, R., et al. (2004). Increased caspase activation in peripheral blood mononuclear cells of patients with Alzheimer's disease. Exp. Neurol. 190, 254-262. doi: 10.1016/j. expneurol.2004.07.009

Thurner, B., Roder, C., Cieckmann, D., Heuer, M., Kruse, M., Glaser, A., et al. (1999). Generation of large numbers of fully mature and stable dendritic cells from leukapheresis products for clinical application. J. Immunol. Methods 223, 1-15. doi: 10.1016/s0022-1759(98)00208-7

Ulitsky, I., and Bartel, D. P. (2013). lincRNAs: genomics, evolution, and mechanisms. Cell 154, 26-46. doi: 10.1016/j.cell.2013.06.020

Wahlestedt, C. (2013). Targeting long non-coding RNA to therapeutically upregulate gene expression. Nat. Rev. Drug Discov. 12, 433-446. doi: 10.1038/ nrd4018

Wang, P., Xue, Y., Han, Y., Lin, L., Wu, C., Xu, S., et al. (2014). The STAT3-binding long noncoding RNA lnc-DC controls human dendritic cell differentiation. Science 344, 310-313.

Wang, X. D., Zheng, M., Lou, H. F., Wang, C. S., Zhang, Y., Bo, M. Y., et al. (2016). An increased prevalence of self-reported allergic rhinitis in major Chinese cities from 2005 to 2011. Allergy 71, 1170-1180. doi: 10.1111/all.12874

Wilfried, P., Lass-Flörl, C., and Wilflingseder, D. (2016). Generation of human monocyte-derived dendritic cells from whole blood. J. Vis. Exp. 118:54968.

Wilson, M. S., Taylor, M. D., Balic, A., Finney, C. A., Lamb, J. R., and Maizels, R. M. (2005). Suppression of allergic airway inflammation by helminth-induced regulatory t cells. J. Exp. Med. 202, 1199-1212. doi: 10.1084/jem.20042572

Yanming, Z., Zhao, Y., Zhang, Y., and Zhang, L. (2019). HLA-II genes are associated with outcomes of specific immunotherapy for allergic rhinitis. Int. Forum. Allergy Rhinol. 9, 1311-1317. doi: 10.1002/alr.22384

Conflict of Interest: The authors declare that the research was conducted in the absence of any commercial or financial relationships that could be construed as a potential conflict of interest.

Copyright (c) 2021 Zhou, Chen, Zheng, Shen, Sun, Yang, Min, Bao, Zhang, Zhao, Wang and Wang. This is an open-access article distributed under the terms of the Creative Commons Attribution License (CC BY). The use, distribution or reproduction in other forums is permitted, provided the original author(s) and the copyright owner(s) are credited and that the original publication in this journal is cited, in accordance with accepted academic practice. No use, distribution or reproduction is permitted which does not comply with these terms. 